\title{
Multi-centric European post-market follow-up study of the Neuroform Atlas Stent System: primary results
}

\author{
Pierre-Henri Lefevre ${ }^{\circ},{ }^{1}$ Peter Schramm, ${ }^{2}$ André Kemmling, ${ }^{3}$ Xavier Barreau, ${ }^{4}$ \\ Gaultier Marnat 이, ${ }^{4}$ Michel Piotin, ${ }^{5}$ Ansgar Berlis, ${ }^{6}$ Isabel Wanke, ${ }^{7}$ Alain Bonafe (1) , \\ Emmanuel Houdart, ${ }^{8}$ ATLAS EU PMCF Investigators
}

Correspondence to Dr Pierre-Henri Lefevre, Neuroradiology department, Hôpital Gui de Chauliac Service de Neuroradiologie, 34295 Montpellier, France; ph. lefevre30@gmail.com

\begin{abstract}
Background Few prospective series have described the safety and effectiveness of the Neuroform Atlas Stent System. We aimed to investigate the efficacy and safety of the device in patients treated for unruptured aneurysm.
\end{abstract}

Methods ATLAS EU PMCF is a consecutive, prospective, multicentric study that included patients with unruptured saccular aneurysm of all sizes. Follow-up visits were scheduled at 3-6 months and 12-16 months with digital subtraction angiography (DSA) or MRI imaging followup as per the site standard of care. The primary efficacy endpoint was adequate aneurysm occlusion (Raymond Roy occlusion grade I and II) on 12 month angiography. The primary safety endpoint was any major stroke or ipsilateral stroke or neurological death within 12 months. Results Of the 106 patients consented, 105 were treated with at least one Neuroform Atlas stent. There was a failed implantation attempt in 1 patient, 85 patients received lateral stenting, and 19 patients received Y-stenting. Mean aneurysm neck size was $4.2 \mathrm{~mm}$ (range 1.9-33 mm). Adequate occlusion was observed in $95.1 \%$ immediately after the procedure and in $98.9 \%$ of cases at 1 year DSA follow-up. Overall, $1.0 \%$ (1/102; $95 \% \mathrm{Cl} 0.0 \%$ to $5.3 \%)$ of patients experienced a primary safety endpoint of major stroke. Three minor strokes resulted in a modified Rankin Scale score of 2. Conclusions In this multicentric, prospective study, stent-assisted coiling of medium size unruptured aneurysms with the Neuroform Atlas stent resulted in a favorable rate of satisfactory occlusion. In our findings, the use of the Y-stenting technique was associated with increased rates of procedural complications.

Clinical trial registration https://clinicaltrials.gov/ct2/ show/NCT02783339.

\section{INTRODUCTION}

Wide-neck or aneurysms with unfavorable morphology have always been a challenge for endovascular treatment. Many improvements in the endovascular field such as the remodeling technique or intracranial stenting have led to endovascular treatment options for most types of aneurysms. ${ }^{12}$ Among these advances, the Neuroform Atlas stent was designed as an open-cell stent to address the challenges with tortuous anatomy. Treatment has been frequently impeded due to improper microcatheter size and limited access to the target aneurysm. Designed to address this, the Neuroform Atlas stent is suitable for a 0.017 inch microcatheter delivery. Initial experiences consisting of mostly retrospective and single centered investigations were published, but limitations were noted due to their study designs and a lack of patients or follow-up. ${ }^{3-13}$ We therefore conducted a prospective and multicentric post-market study to determine the clinical and technical outcomes following treatment of intracranial aneurysms with the Neuroform Atlas stent.

\section{METHODS}

\section{Study design}

The European Atlas post-market clinical follow-up study (ATLAS EU PMCF) was a prospective, observational, single arm, multicentric cohort which included patients with unruptured or previously ruptured ( $\geq 3$ months) intracranial aneurysms. Each investigating center obtained institutional review board or ethics committee approval. Patients were enrolled between October 2016 and February 2018 at 11 hospital centers.

The primary safety endpoint was the permanent morbidity and mortality rate at 1 year postprocedure, defined as any neurological death or major ipsilateral stroke leading to an increase of $\geq 4$ points in the National Institutes of Health Stroke Scale (NIHSS) at 24 hours and associated worsening of the modified Rankin Scale $(\mathrm{mRS}) \geq 3$ or change from baseline by 2 points at 90 days post-event. A stroke with clinical symptoms that resolved within 24 hours and with brain imaging showing infarction of vascular origin was considered a minor stroke.

Secondary endpoints were designed to provide information about effectiveness and angiographic results of the Neuroform Atlas Stent System and were as follows:

- Procedural technical success (accurate stent delivery, accurate positioning)

- Aneurysm occlusion status (Raymond Roy score) assessed at 1 year (12 to 16 months) post-procedure follow-up by digital subtraction angiography (DSA)

- In-stent stenosis ( $>50 \%$ of the arterial diameter)

- Incidence of retreatment occurring 12 to 16 months post-procedure.

Secondary safety endpoints were studied, such as the rates of all intraprocedural adverse events and device malfunctions, symptomatic and asymptomatic adverse events that occurred during follow-up, subarachnoid hemorrhage $(\mathrm{SAH})$, aneurysm rupture/re-rupture rate, new or worsening 
major ipsilateral stroke measured by NIHSS and mRS, and the duration between the index procedure and the occurrence of major ipsilateral stroke or death.

\section{Inclusion and exclusion criteria}

Patients aged 18 years or older harboring an unruptured or previously ruptured ( $\geq 3$ months) intracranial aneurysm in the anterior and posterior circulation treated with the Neuroform Atlas stent were eligible to enroll in this study. All patients provided a signed informed consent in order to participate. Key exclusion factors included patients with an $\mathrm{mRS} \geq 3$, previously ruptured target aneurysm $\leq 3$ months before, fusiform aneurysms, multiple aneurysms (except adjacent aneurysms being treated by the same stent), aneurysms associated with an arteriovenous malformation or potential hemorrhagic complications, and aneurysms previously treated with stenting other than the Neuroform Atlas Stent System. Pregnant or child-feeding patients and those with contraindications to MR imaging, nitinol (due to allergy), heparin, clopidogrel or aspirin were also excluded.

\section{Endovascular procedures}

Each procedure was performed via a femoral approach with the patient under general anesthesia. Most of the patients received an initial heparin bolus (70-100 UI/ $\mathrm{kg}$ ) followed by continuous infusion (targeted activated clotting time between 250-300 s). Antiplatelet drugs were administered under the direction and management of each participating investigator according to each institution's standard of care protocol. The aneurysm and the parent vessel were analyzed via angiography before stent placement.

Patients were treated with the Neuroform Atlas Stent System using various coiling techniques. The jailing technique that secured the microcatheter between the stent and the vessel wall was used in $60.9 \%(64 / 105)$ of cases, insertion of the microcatheter through the stent struts (eg, recrossing) was used in $32.4 \%$ $(34 / 105)$ of cases, and post-coiling was used in the remaining $6.7 \%(7 / 105)$ of cases.

\section{Clinical evaluations}

Clinical evaluations were performed before and after the index procedure, before hospital discharge, at 3-6 months postprocedure, and 12-16 months post-procedure. Neurological status and mRS were compared with initial baseline measures for indicators of clinical improvement or worsening. All the clinical events were classified according to a written prespecified protocol and adjudicated by an independent clinical events committee (CEC).

\section{Radiological evaluation}

An independent core laboratory reviewed all the images via a central database. The modified Raymond Roy scale was used to classify the occlusion as complete, residual neck, or residual aneurysm based on the working projections. ${ }^{10}$ Radiological follow-up was collected at 3-6 months and 12-16 months postprocedure per institutional standards. The occlusion status was compared with post-procedural results to determine the rates of progressive occlusion defined as any perceptible improvement from the initial results. Recurrence was classified as minor or major and the latter was defined as a requirement of subsequent treatment.

\section{Clinical characteristics}

Of the 106 consented patients, 105 patients with successful implantation of one or more Neuroform Atlas stents were reported, as shown in online supplemental figure 1. Patients who were consented, enrolled, and successfully received a stent device implant were counted as the modified intention to treat (mITT) group. Of the mITT group patients, 79 (75.2\%) were female with a mean age of 55.3 years (range $28-81$ years). Twenty-five $(23.8 \%)$ patients reported having a previous non-target aneurysm, of which $11(10.5 \%)$ reported previous rupture.

\section{Pre-procedure medication usage}

No antiplatelet protocol was prespecified in this study. Treatments were given according to the local institutional standard of care protocol and at the discretion of the physician investigator. Most of the patients $(61.9 \% ; 65 / 105)$ benefited from dual antiplatelet therapy (DAPT) administered pre-procedure, consisting of aspirin and one or more $\mathrm{P}_{2} \mathrm{Y}_{12}$ inhibitors. Among them, $46(43.8 \%)$ received clopidogrel, 19 (18.1\%) received ticagrelor, three $(2.9 \%)$ received prasugrel, two $(1.9 \%)$ received clopidogrel + prasugrel, and one (1.0\%) received clopidogrel + ticagrelor.

\section{RESULTS}

\section{Characteristics of target aneurysms}

The majority of aneurysms treated were between $4-7 \mathrm{~mm}$ in diameter $(55.2 \% ; 58 / 105)$. Most treated aneurysms had a wide neck with a dome-to-neck ratio lower than 1.5 (57.1\%; 60/105). The shape of the treated aneurysm was spherical in most cases (46.7\%; 49/105). The mean aneurysm size was $4.9 \mathrm{~mm}$ (range $1.8-17 \mathrm{~mm}$ ) and the mean neck width was $4.2 \mathrm{~mm}$ (range 1.9-33 $\mathrm{mm})$. The target aneurysm had been previously treated in 19 (18\%) patients. Anterior circulation target aneurysm locations represented $90.5 \%$ (95/105) of cases. The most common location was the middle cerebral artery (MCA), seen in 40 patients (38\%). Details regarding target aneurysm characteristics are included in table 1.

\section{Technical results}

The overall reported procedure technical success was $94.7 \%$ (124/131). The reported accurate stent positioning was $94.7 \%$ $(124 / 131)$ and accurate delivery was 96.9\% (127/131). The median procedure duration was 95 min (range 35-375 min). All patients received general anesthesia (100\%; 105/105).

In descending order and when analyzed per patient, the number of stents most commonly used was one device $(78.1 \%$; $82 / 105)$, two devices $(20.0 \% ; 21 / 105)$, three devices $(1.0 \%$; $1 / 105)$, and four devices $(1.0 \% ; 1 / 105)$. Similarly, for number of stents successfully implanted on a per patient level, patients received one device $(81.0 \% ; 85 / 105)$, two devices $(17.1 \%$; $18 / 105)$, or three devices $(1.9 \% ; 2 / 105)$. In procedures utilizing more than one stent, $90.5 \%(19 / 21)$ utilized Y-stenting and in two other cases additional stenting techniques were needed. The median coil packing density was $30.1 \%$ and the mean was $47.1 \pm 31.1 \%$. Detailed technical results are available online (see online supplemental material).

\section{Permanent morbidity and mortality}

Primary safety endpoint findings in relation to morbidity and mortality as adjudicated by the CEC, including ipsilateral strokes, are included in table 2.

There was an overall device complication rate of 5.7\% (6/105) through 1 year follow-up: there was one peri-procedural vascular stent thrombosis and five events were recorded (four in the postprocedural days 1-180, one of late occurrence after day 181). 


\begin{tabular}{|c|c|}
\hline Aneurysm characteristics & Results \\
\hline \multicolumn{2}{|l|}{ Aneurysm neck width (mm) } \\
\hline Mean $\pm S D(N)$ & $4.2+3.1(105)$ \\
\hline Median (Q1-Q3) & $3.8(3-4.5)$ \\
\hline Range (min-max) & $31.1(1.9-33)$ \\
\hline \multicolumn{2}{|l|}{ Aneurysm size $(\mathrm{mm})^{*}$} \\
\hline Mean \pm SD (N) & $5.8 \pm 2.5(105)$ \\
\hline Median (Q1-Q3) & $5.3(4-6.7)$ \\
\hline Range (min-max) & $14.5(2.5-17)$ \\
\hline \multicolumn{2}{|l|}{ Aneurysm size by categories $(\mathrm{mm})^{*}$} \\
\hline$<4 \mathrm{~mm}$ & $21.0 \%(22 / 105)$ \\
\hline$\geq 4 \mathrm{~mm}$ and $<7 \mathrm{~mm}$ & $55.2 \%(58 / 105)$ \\
\hline$\geq 7 \mathrm{~mm}$ and $<10 \mathrm{~mm}$ & $19.0 \%(20 / 105)$ \\
\hline$\geq 10 \mathrm{~mm}$ & $4.8 \%(5 / 105)$ \\
\hline \multicolumn{2}{|l|}{ Dome/neck ratio } \\
\hline$<1.5$ & $57.1 \%(60 / 105)$ \\
\hline \multicolumn{2}{|l|}{ Aneurysm discovery } \\
\hline Incidental & $77.4 \%(82 / 105)$ \\
\hline Symptomatic & $4.7 \%(5 / 105)$ \\
\hline Previously ruptured & $12.4 \%(13 / 105)$ \\
\hline Recanalization & $5.7 \%(6 / 105)$ \\
\hline Anterior location & $90.5 \%(95 / 105)$ \\
\hline Anterior cerebral artery (ACA) & $13.3 \%(14 / 105)$ \\
\hline Anterior communication artery (ACoM) & $14.3 \%(15 / 105)$ \\
\hline Internal carotid artery (ICA) & $24.8 \%(26 / 105)$ \\
\hline Middle cerebral artery (MCA) & $38.1 \%(40 / 105)$ \\
\hline Posterior location & $9.5 \%(10 / 105)$ \\
\hline Basilar artery (BA) & $4.8 \%(5 / 105)$ \\
\hline Posterior cerebral artery (PCA) & $2.9 \%(3 / 105)$ \\
\hline Posterior inferior cerebral artery (PICA) & $1.0 \%(1 / 105)$ \\
\hline Vertebrobasilar (VB) & $1.0 \%(1 / 105)$ \\
\hline
\end{tabular}

*Aneurysm size calculated from the maximum of dome height, dome width and dome depth. mITT, modified intention to treat.

Site-reported technical results and perioperative information is provided in online supplemental table 1.

\section{Major and minor strokes}

Overall, there were five $(4.8 \%)$ and eight $(7.6 \%)$ patients who experienced major and minor ipsilateral strokes, respectively. When adjusting for major ipsilateral strokes per NIHSS criteria only, there were five major ipsilateral strokes reported with three of them being device-related and occurring during the peri-procedural treatment time. Among the device-related major strokes, there were two deemed to be of thrombotic causes and one was due to vessel perforation. Of the five major ipsilateral strokes, there were two which occurred later at day 46 and day 60 post-procedure.

There was one major stroke reported as medication-related, which was due to an improper withdrawal from clopidogrel by the patient.

One case was noted as vessel perforation with subsequent parenchymal hemorrhage, potentially due to difficulty navigating the microcatheter. Post-procedure angiogram showed contrast leak in the left parietal area which was confirmed by CT demonstrating a temporal SAH before the patient expired. The case was recorded as a device misplacement during the procedure and was deemed to be unrelated to the device delivery system, but related to the index procedure. This case met the primary safety endpoint criteria per ATLAS EU PMCF definition.

\section{Minor strokes}

There were eight minor ipsilateral strokes reported and four were device related. Of the device-related minor strokes, three were also deemed to be related to the index procedure. Two minor strokes were attributed solely to the index procedure. One minor stroke was related to medication with unknown relationship to the device. The final minor stroke was related to a pre-existing condition.

\section{Deaths}

Two patients died during the study, one from a pulmonary carcinoma and one following procedure difficulties to navigate the microcatheter, which led to vessel perforation resulting in $\mathrm{SAH}$ and neurological death. Both deaths were deemed unrelated to the stent device and delivery system according to CEC adjudication. The latter patient death was deemed to be related to the index procedure.

\section{Other complications}

Apart from intracranial complications, two (1.9\%) patients had a puncture site hematoma and two (1.9\%) patients experienced a contrast encephalopathy. No patients in the study were observed to have target aneurysm ruptures through 1 year post-procedure in the mITT population, as confirmed by the CEC.

\section{Post-procedure medication}

A majority of patients $(88.6 \%$; 93/105) benefited from DAPT therapy at the time of discharge, consisting of aspirin and one or more P2Y ${ }_{12}$ inhibitors. Among them, 61 (58.1\%) received clopidogrel, 27 (25.7\%) received ticagrelor, eight (7.6\%) received prasugrel, and three received clopidogrel + prasugrel.

\section{Occlusion status}

Per core lab assessment, complete occlusion as defined by the Raymond Roy Occlusion Classification class I, was seen in 91.3\% in the perioperative period. Complete occlusion was seen in $97.4 \%$ and $90.3 \%$ of patients at 3-6 month and 12-16 month follow-up visits, respectively. Angiographic findings regarding occlusion status are summarized in table 3 .

Table 2 CEC-adjudicated permanent morbidity and mortality (major ipsilateral stroke or neurological death through 1 year) (mITT)

\begin{tabular}{llll}
\hline Analysis & $\begin{array}{l}\text { \% Subject (n/N) } \\
\text { (\# event) }\end{array}$ & 95\% Cl* & P value \\
\hline $\begin{array}{l}\text { Permanent morbidity and mortality } \\
\text { (major ipsilateral stroke or neurological } \\
\text { death) }\end{array}$ & $1.0 \%(1 / 102)$ & $0.0 \%$ to $5.3 \%$ & $<0.001$ \\
\hline Neurologic death & $1.0 \%(1 / 102)$ & $0.0 \%$ to $5.3 \%$ & \\
\hline Major ipsilateral stroke & $1.0 \%(1 / 102)$ & $0.0 \%$ to $5.3 \%$ & \\
\hline Major ipsilateral stroke per NIHSS & $4.9 \%(5 / 102)$ & $1.6 \%$ to $11.1 \%$ & \\
\hline Major ipsilateral stroke per mRS & $1.0 \%(1 / 102)$ & $0.0 \%$ to $5.3 \%$ & \\
\hline Worst case scenario§ & $3.8 \%(4 / 105)$ & $1.0 \%$ to $9.5 \%$ & $<0.001$ \\
\hline Best case scenario & $1.0 \%(1 / 105)$ & $(0.0 \%, 5.2 \%)$ & $<0.001$ \\
\hline *Clopper-Pearson & & &
\end{tabular}

${ }^{*}$ Clopper-Pearson exact confidence interval.

tNIHSS decline $\geq 4$ points lasting 24 hours or more.

$\neq \mathrm{mRS} \geq 3$ at a minimum of 90 days post-event.

$\S$ Missing endpoint data were imputed as failure.

CEC, clinical events committee ; mITT, modified intention to treat; mRS, modified Rankin Scale; NIHSS, National Institutes of Health Stoke Scale. 


\begin{tabular}{|c|c|c|c|}
\hline & Post-procedure* & 3-6 months* & 1 Year* \\
\hline $\begin{array}{l}\text { Complete } \\
\text { occlusion } \\
\text { Raymond Roy I }\end{array}$ & $91.3 \%(94 / 103)$ & $97.4 \%$ (37/38) & $90.3 \%(84 / 93)$ \\
\hline $\begin{array}{l}\text { Aneurysm neck } \\
\text { Raymond Roy II }\end{array}$ & $3.9 \%(4 / 103)$ & $0.0 \%(0 / 38)$ & $8.6 \%(8 / 93)$ \\
\hline $\begin{array}{l}\text { Incomplete } \\
\text { occlusion } \\
\text { Raymond Roy III }\end{array}$ & $4.9 \%(5 / 103)$ & $2.6 \%(1 / 38)$ & $1.1 \%(1 / 93)$ \\
\hline $\begin{array}{l}\text { Adequate } \\
\text { occlusion } \\
\text { Raymond Roy } \\
\text { I or II }\end{array}$ & $95.1 \%(98 / 103)$ & $97.4 \%$ (37/38) & $98.9 \%(92 / 93)$ \\
\hline
\end{tabular}

\section{Parent artery stenosis}

Among the 93 patients who had an imaging follow-up at 1 year, $63(67.7 \%)$ had no or lower than $25 \%$ parent artery stenosis, six $(6.5 \%)$ were between $25 \%$ and $50 \%$, and none were greater than $50 \%$. The remaining $24(25.8 \%)$ patients were not assessed due to inaccessible records. One year follow-up imaging was undertaken per institutional standard of care. When MR angiography or CT angiography were the chosen modalities, the aneurysmal occlusion was core lab assessable but the stenosis status was not.

\section{Modified Rankin Scale}

At 12-16 month follow-up, 73 (76.0\%) patients had an mRS of $0,18(18.8 \%)$ had an mRS of 1 , and three $(3.1 \%)$ had an mRS of 2 . There were two $(2.1 \%)$ patient deaths, one related to the intervention and one related to another medical condition (cancer), which were deemed to be unrelated to the device implant or delivery system and which were counted as an mRS of 6. Observed changes in reported $\mathrm{mRS}$ evaluations are displayed in table 4.

\section{DISCUSSION}

This multicentric prospective study included elective aneurysmal treatment with the Neuroform Atlas stent and is the largest of its kind to date in Europe. This study demonstrated a high technical success rate with an accurate delivery reported as high as $96.9 \%$. This rate is similar to current low-profile stents such as the LVIS Jr and Leo Baby. ${ }^{13-15}$

Adequate occlusion, as defined by grade I and II according to the Raymond Roy classification, was seen in $98.9 \%$ of the 93 patients that completed the follow-up with DSA. This result is

\begin{tabular}{|c|c|c|c|}
\hline mRS score & Baseline & $\begin{array}{l}\text { 3-6 month follow-up } \\
\%(n / N)^{*}\end{array}$ & $\begin{array}{l}12-16 \text { month } \\
\text { follow-up } \\
\%(n / N) \dagger\end{array}$ \\
\hline 0 & $81.0 \%(85 / 105)$ & $76.2 \%(77 / 101)$ & $76.0 \%(73 / 96)$ \\
\hline 1 & $17.1 \%(18 / 105)$ & $18.8 \%(19 / 101)$ & $18.8 \%(18 / 96)$ \\
\hline 2 & $1.9 \%(2 / 105)$ & $4.0 \%(4 / 101)$ & $3.1 \%(3 / 96)$ \\
\hline 6 & & $1.0 \%(1 / 101)$ & $2.1 \%(2 / 96)$ \\
\hline
\end{tabular}

slightly higher than other studies and likely attributable to the longer post-procedure follow-up protocol as compared with other investigations. ${ }^{8121316}$ The additional composite primary efficacy endpoint of complete aneurysm occlusion (Raymond Roy I) without significant (>50\%) parent artery stenosis or aneurysm retreatment was achieved in $89.9 \%$ (95\% CI $80.2 \%$ to $95.8 \%$ ) of patients.

The completion rate of follow-up assessments was high with only three patients lost to follow-up (LTFU) and 93 patients $(88.5 \%)$ completing vascular imaging at 12 months. The latter is significantly higher than that reported in previous published investigations. $^{58121316}$

The rate of permanent morbidity and mortality was $1.0 \%$ $(1 / 105 ; \mathrm{p}<0.001,95 \%$ CI $0.0 \%$ to $5.2 \%)$ in the best-case analysis. Regarding morbidity and mortality findings and devicerelated events, there was one major ipsilateral stroke per safety endpoint criteria and three minor strokes resulting in an mRS score of 2. Alternatively, the rate of permanent morbidity and mortality was estimated at 3.8\% (4/105, p $<0.001,95 \% \mathrm{CI}$ $1.0 \%, 9.5 \%)$ in the worst-case analysis, accounting for three LTFU patients. This rate seems higher than previously published series including only unruptured aneurysms. ${ }^{49131718}$ This relatively higher rate is likely attributable to the more comprehensive follow-up (every event was tracked and counted), the lack of a prespecified antiplatelet protocol, and the relatively high proportion of Y-stenting procedures.

There were 19 patients included in the present analysis who were treated with Y-stenting. Our experience in the ATLAS EU PMCF study supports the notion that open cell stenting, such as with the Neuroform Atlas Stent System, is particularly suitable for this kind of technical approach, allowing treatment of wide neck aneurysms through a 0.017 inch catheter. Nevertheless, it has been extensively reported that this technique suffers from a higher rate of morbidity. ${ }^{19}$ Ciccio et al reported recently up to $12.7 \%$ of symptomatic complications in 55 patients treated with Y-stenting which was associated with a permanent neurological morbidity rate of $3.6 \%{ }^{17}$ Similar results were shared by Aydin et al who reported a $3.3 \%$ permanent neurological morbidity rate among 30 patients treated with Y-stenting. ${ }^{3}$ Contrary to other published investigations ${ }^{712} 13$ which included smaller quantities of Y-stenting procedures, our study involving the use of open cell stents reported a low morbidity rate of $1 \%$ within the $18 \%$ of study patients treated with Y-stenting. However, the only patient who died secondary to intracranial perforation was also treated with Y-stenting.

No patient experienced a parent artery stenosis $>50 \%$ and only one stent thrombosis was reported. This result is significantly lower than other published reports with braided stents. ${ }^{20}$ It has been postulated that a braided design is slightly more thrombogenic and the present work may support this hypothesis. ${ }^{21}$

Compared to the American literature, ${ }^{7} 1222$ we observed a higher proportion of MCA aneurysms (38.1\%) and lower mean diameter in the ATLAS EU PMCF study. There was 21\% of patients who had an aneurysm diameter lower than $4 \mathrm{~mm}$. Hence, this could partially explain the higher reported rate of occlusion during follow-up as previously shown by Caragliano et $a l$ in a similar Italian registry study. ${ }^{23}$ Comparison with a similar prospective trial shows that our morbidity rate is comparable with $4.8 \%$ of patients experiencing major stroke as compared with $4.4 \%$ in the Atlas IDE trial. ${ }^{23}$ In the Atlas IDE trial, two stents were used in $15.9 \%$ of patients, which is comparable to the proportion of Y-stenting in our cohort. Interestingly, even though aneurysms lower than $4 \mathrm{~mm}$ were excluded from the Atlas IDE trial, efficacy rates regarding occlusion were very 
similar to ours with $96.1 \%$ of Raymond Roy I and II as compared with $98.9 \%$ in the present study. Recently, a sub-analysis from the ATLAS IDE data about the MCA aneurysm treatment was published. ${ }^{24}$ Since ATLAS EU contains more MCA aneurysms, sub-analysis from this dataset will be undertaken in the future.

Stent-assisted coiling (SAC) for small and medium size (4 to $9 \mathrm{~mm}$ ) wide neck unruptured aneurysms is a competitive technology compared with intrasaccular devices and flow diverters. The SAC and the intrasaccular devices are prone to recanalization but retreatment appears easier after SAC procedures. ${ }^{25}$ Flow diverters may carry the risk of side branch occlusion in the long term. $^{26}$

We acknowledge that this study suffers several limitations such as the non-randomized design, which could include a selection bias and the lack of a control group with a different stent device. Likewise, no antiplatelet protocol was agreed before inclusion, therefore each center protocol was localized. This resulted in different results among centers in terms of drug usage and dosage, but also reflects 'real life' usage of such products.

\section{CONCLUSION}

The ATLAS EU PMCF study is the first multicentric and prospective study regarding the Neuroform Atlas Stent System in Europe. The results show that the Neuroform Atlas stent is a relatively safe and very efficient method to treat unruptured saccular aneurysms of all sizes.

\author{
Author affiliations \\ ${ }^{1}$ Neuroradiology department, Gui de Chauliac Hospital Montpellier University \\ Hospital, Montpellier, France \\ ${ }^{2}$ Department of Neuroradiology, University Medical Center Schleswig-Holstein, \\ Luebeck, Germany \\ ${ }^{3}$ Institute of Neuroradiology, University Medical Center Schleswig-Holstein, Luebeck, \\ Germany \\ ${ }^{4}$ Interventional and Diagnostic Neuroradiology, Bordeaux University Hospital, \\ Bordeaux, France \\ ${ }^{5}$ Department of Interventional Neuroradiology, Fondation Rothschild Hospital, Paris, \\ France \\ ${ }^{6}$ Department of Diagnostic and Interventional Neuroradiology, Klinikum Augsburg, \\ Augsburg, Bayern, Germany \\ ${ }^{7}$ Neuroradiology Department, Hirslanden Clinic, Zurich, Switzerland \\ ${ }^{8}$ Neuroradiology Department, Lariboisière Hospital, Paris, France
}

Collaborators ATLAS EU PMCF Investigators and coordinators: Ansgar Berlis, Christoph Maurer, Martin Seidler, Ilse Kummer, Sabine Hanisch, Gabriele JochumPfeilsticker, Marius Hartmann, Steffen Reissberg, Sascha Schiffler, Nadja Rieckehr, Werner Weber, Annika Kowoll, Anushe Weber, Sebastian Fischer, Mara Postert, Peter Schramm, Thomas Eckey, André Kemmling, Alexander Neumann, Ramona Schramm, Alain Bonafé, Vincent Costalat, Carlos Riquelme, Pierre-Henri Lefevre, Grégory Gascou, Marinette Moynier, Emmanuel Houdart, Vittorio Civelli, Alexis Guédon, JeanPierre Saint-Maurice, Marc-Antoine Labeyrie, Alexandre Parpaleix, Baptiste Bonnet, Kamil Zelenak, Jana Zelenakova, Branislav Kolarovszki, Michel Piotin, Raphael Blanc, Stanislas Smajda, Jean-Philippe Desilles, Gabriele Ciccio, Robert Fahed, Hocine Redjem, Mylène Hamdani, Malek Ben Maacha, Lucia Lopes, Xavier Barreau, Gaultier Marnat, Florent Maire, Marine Premont, Laura Albizu, Jean-Christophe Gentric, Julien Ognard, Géraldine Viard, Ana Paula Narata, Vladimir Charron, Nicolas Charron

Contributors The last two authors contributed equally to the manuscript. All authors made substantial contributions to the conception and design, analysis, and interpretation of data; drafted or critically revised the article; and gave final approval of the version to be published.

Funding The ATLAS EU PMCF study (Evaluation of Safety and Performance of the Neuroform Atlas ${ }^{\mathrm{TM}}$ Stent System for Intracranial Aneurysm Treatment - Post Market Clinical Follow-up) was funded by Stryker Neurovascular (Fremont, California, United States)

Competing interests P-HL reports paid lectures with Stryker, Medtronic and Phenox outside the present study. PS reports speaker honoraria and travel support from Penumbra, Phenox and Stryker outside the present study. AK reports consulting and proctoring activities with Phenox, Penumbra, Stryker, Acandis outside the present study. XB reports service and related fees with Stryker outside this study and within past 3 years. Proctored or received service-related fees from MicroVention (Proctor). GM reports paid lectures with Microvention and Medtronic. MP reports shares in Basecamp Vascular. ABe reports a proctoring activity for Stryker outside this study and within past 3 years, proctored or received service-related fees from MicroVention (Proctor), Sequent Medical (Proctor), Phenox (lecture honorary, CEC), and Penumbra (lecture honorary). IW is speaker for Bayer, not related to the product. ABo is consultant for Stryker, Balt and Phenox. EH is the principal investigator of the present ATLAS EU study.

\section{Patient consent for publication Not required.}

Ethics approval The study was performed in accordance with International Standard ISO 14155, Declaration of Helsinki, Good Clinical Practice (GCP), International Conference on Harmonization ( $\mathrm{ICH}$ ), and/or any other applicable guidelines and applicable local regulations, whichever affords greater protection to the patient. Adverse event data were collected and analyzed per MDD 93/42/EEC and MEDDEV 2.12.1 guidelines.

\section{ORCID iDs}

Pierre-Henri Lefevre http://orcid.org/0000-0002-6196-3869

Gaultier Marnat http://orcid.org/0000-0002-7611-7753

Alain Bonafe http://orcid.org/0000-0002-9187-1375

\section{REFERENCES}

1 King B, Vaziri S, Singla A, et al. Clinical and angiographic outcomes after stentassisted coiling of cerebral aneurysms with enterprise and Neuroform stents: a comparative analysis of the literature. J Neurointerv Surg 2015;7:905-9.

2 Moret J, Cognard C, Weill A, Castaings L, et al. [Reconstruction technic in the treatment of wide-neck intracranial aneurysms. Long-term angiographic and clinical results. Apropos of 56 cases]. J Neuroradiol 1997;24:30-44.

3 Aydin K, Balci S, Sencer S, et al. Y-stent-assisted coiling with low-profile Neuroform Atlas stents for endovascular treatment of wide-necked complex intracranial bifurcation aneurysms. Neurosurgery 2020;87:744-53.

4 Baek JW, Jin S-C, Kim JH, Yoo MW, et al. Initial multicentre experience using the Neuroform Atlas stent for the treatment of un-ruptured saccular cerebral aneurysms. Br J Neurosurg 2020;34:333-8.

5 Cay F, Peker A, Arat A. Stent-assisted coiling of cerebral aneurysms with the Neuroform Atlas stent. Interv Neuroradiol 2018;24:263-9.

6 Goertz L, Dorn F, Siebert E, et al. Safety and efficacy of the Neuroform Atlas for stentassisted coiling of intracranial aneurysms: a multicenter experience. J Clin Neurosci 2019;68:86-91.

7 Gross BA, Ares WJ, Ducruet AF, et al. A clinical comparison of atlas and LVIS jr stentassisted aneurysm coiling. J Neurointerv Surg 2019;11:171-4.

$8 \mathrm{Kim}$ CH, Kim YH, Sung SK, et al. Clinical safety and effectiveness of stent-assisted coil embolization with Neuroform Atlas stent in intracranial aneurysm. J Korean Neurosurg Soc 2020;63:80-8.

9 Quintana EM, Valdes PV, Deza EM, et al. Initial experience and one-year follow-up with Neuroform Atlas stent system for the treatment of brain aneurysms. Interv Neuroradiol 2019;25:521-9.

10 Raymond J, Guilbert F, Weill A, et al. Long-term angiographic recurrences after selective endovascular treatment of aneurysms with detachable coils. Stroke 2003;34:1398-403.

11 Ten Brinck MFM, de Vries J, Bartels RHMA, et al. Neuroform Atlas stent-assisted coiling: preliminary results. Neurosurgery 2019;84:179-89.

12 Tsai JP, Hardman J, Moore NZ, et al. Early post-humanitarian device exemption experience with the Neuroform Atlas stent. J Neurointerv Surg 2019;11:1141-4.

13 Ulfert C, Pham M, Sonnberger M, et al. The Neuroform Atlas stent to assist coil embolization of intracranial aneurysms: a multicentre experience. J Neurointerv Surg 2018;10:1192-6.

14 Aydin K, Arat A, Sencer S, et al. Stent-assisted coiling of wide-neck intracranial aneurysms using low-profile Leo baby stents: initial and midterm results. AJNR Am J Neuroradio/ 2015;36:1934-41.

15 Grossberg JA, Hanel RA, Dabus G, et al. Treatment of wide-necked aneurysms with the low-profile visualized intraluminal support (LVIS jr) device: a multicenter experience. J Neurointerv Surg 2017;9:1098-102. 
16 Zhao B, Yin R, Lanzino G, et al. Endovascular coiling of wide-neck and wide-neck bifurcation aneurysms: a systematic review and meta-analysis. AJNR Am J Neuroradiol 2016;37:1700-5.

17 Ciccio G, Robert T, Smajda S, et al. Double stent assisted coiling of intracranial bifurcation aneurysms in $Y$ and $X$ configurations with the Neuroform Atlas stent: immediate and mid term angiographic and clinical follow-up. J Neurointerv Surg 2019;11:1239-42.

18 Jankowitz BT, Hanel R, Jadhav AP, et al. Neuroform Atlas stent system for the treatment of intracranial aneurysm: primary results of the Atlas humanitarian device exemption cohort. J Neurointerv Surg 2019;11:801-6.

19 Granja MF, Cortez GM, Aguilar-Salinas P, et al. Stent-assisted coiling of cerebral aneurysms using the Y-stenting technique: a systematic review and meta-analysis. J Neurointerv Surg 2019;11:683-9.

20 Park S-Y, Oh J-S, Oh H-J, et al. Safety and efficacy of low-profile, self-expandable stents for treatment of intracranial aneurysms: initial and midterm results - a systematic review and meta-analysis. Interv Neurol 2017;6:170-82.
21 Cagnazzo F, Cappucci M, Lefevre P-H, et al. Treatment of intracranial aneurysms with self-expandable braided stents: a systematic review and meta-analysis. AJNR Am J Neuroradiol 2018;39:2064-9.

22 Zaidat 00, Hanel RA, Sauvageau EA, et al. Pivotal trial of the Neuroform Atlas stent for treatment of anterior circulation aneurysms: one-year outcomes. Stroke 2020;51:2087-94.

23 Caragliano AA, Papa R, Pitrone A, Limbucci N, et al. The low-profile Neuroform Atlas stent in the treatment of wide-necked intracranial aneurysms - immediate and midterm results: an Italian multicenter registry. J Neuroradiol 2020;47:421-427.

24 Hanel RA, Yoon N, Sauvageau E, et al. Neuroform Atlas stent for treatment of middle cerebral artery aneurysms: 1-year outcomes from Neuroform Atlas stent pivotal trial. Neurosurgery 2021;89:102-8.

25 Zhang S-M, Liu L-X, Ren P-W, et al. Effectiveness, safety and risk factors of Woven EndoBridge device in the treatment of wide-neck intracranial aneurysms: systematic review and meta-analysis. World Neurosurg 2020;136:e1-23.

26 Cagnazzo F, Mantilla D, Lefevre P-H, et al. Treatment of middle cerebral artery aneurysms with flow-diverter stents: a systematic review and meta-analysis. AJNR Am J Neuroradiol 2017;38:2289-94. 